\title{
In situ bioassay chambers and procedures for assessment of sediment toxicity with Chironomus riparius
}

\author{
Bruno B. Castro ${ }^{\mathrm{a}, \mathrm{b}}$, Lúcia Guilhermino ${ }^{\mathrm{b}, \mathrm{c}}$, Rui Ribeiro ${ }^{\mathrm{a}, *}$ \\ ${ }^{a}$ Instituto do Ambiente e Vida, Departamento de Zoologia da Universidade de Coimbra, Largo Marquês de Pombal, 3004-517 Coimbra, Portugal \\ ${ }^{\mathrm{b}}$ Centro Interdisciplinar de Investigação Marinha e Ambiental, Rua do Campo Alegre $n^{\circ}$ 823, 4150-180 Porto, Portugal \\ ' Instituto de Ciências Biomédicas de Abel Salazar, Departamento de Estudos de Populações, Laboratório de Ecotoxicologia, \\ Largo Abel Salazar n², 4099-003 Porto, Portugal
}

Received 10 March 2001; accepted 14 March 2003

"Capsule": In situ bioassays are cost-effective and more ecologically relevant tools for sediment toxicity assessment.

\begin{abstract}
The purpose of this study was to develop an in situ sediment bioassay chamber and respective procedures, suitable for performing toxicity bioassays with benthic invertebrates, using the midge Chironomus riparius. It was also our objective to compare the responses obtained under controlled conditions (laboratory 10-day larval growth and survival test) with those obtained in situ. Clean sand and a formulated sediment were incorporated in the in situ bioassay, along with local sediments, as a way of minimizing natural variability due to physicochemical differences among sediments or due to interactions with indigenous organisms. Recovery of organisms was good ( $80-100 \%$ in the control and reference site), indicating that the developed chamber and protocol were suitable for exposing and retrieving $C$. riparius in situ. Results also showed differences between responses obtained with formulated and natural sediment in situ, as well as between laboratory and in situ.
\end{abstract}

(C) 2003 Elsevier Science Ltd. All rights reserved.

Keywords: In situ bioassay; Sediment toxicity; Ecological relevance; Chironomus riparius; Formulated sediment

\section{Introduction}

Risk assessment studies for contaminated sites involve standardized bioassays, which are performed under controlled conditions. However, this laboratory toxicity testing does not always generate ecologically relevant information for the area of concern (Giesy and Hoke, 1989), mainly because field situations may not be accurately simulated in the laboratory, and sample collection, storage or handling can affect sample toxicity, which is particularly relevant in sediment toxicity testing (Burton, 1991; Crane et al., 1995; Chappie and Burton, 1997; Beiras et al., 1998). In situ bioassays are an effective tool to overcome this problem, since they reduce the artifacts related to sample handling and, at the same time, allow a much more realistic exposure (Burton,

\footnotetext{
* Corresponding author. Tel.: + 351-239-822241; fax: + 351-239824226.

E-mail address: rui.ribeiro@zoo.uc.pt (R. Ribeiro).
}

1991; Chappie and Burton, 1997; Tucker and Burton, 1999).

Nebeker et al. (1984) first proposed the idea of in situ sediment test-chambers. Since then, several in situ approaches have been published (e.g. Crane et al., 1995; Monson et al., 1995; Ireland et al., 1996; Shaw and Manning, 1996; Chappie and Burton, 1997). Recently, in situ bioassays have successfully been performed with cladocerans (Pereira et al., 1999), amphipods (DeWitt et al., 1999; Schulz and Liess, 1999; Tucker and Burton, 1999), chironomids (Sibley et al., 1999; Tucker and Burton, 1999; Crane et al., 2000), oligochaetes (Sibley et al., 1999), and bivalves (Soucek et al., 2000). An important feature of these recent field studies is that they used sediment bioassays or focused, in some way, on the importance of including sediments in toxicity bioassays. De Witt et al. (1999) thoroughly discussed the applications and cautions regarding in situ sediment toxicity bioassays.

The present study aimed to (1) develop a cost-effective test-chamber and protocols suitable for in situ sediment 
toxicity testing with larvae of the benthic macroinvertebrate Chironomus riparius Meigen, and (2) to evaluate the ecological relevance of the standardized 10-day larval growth and survival test protocol in estimating the toxicity of sediments, through the comparison of laboratory and in situ results. This latter phase was performed in an acid mine drainage impacted aquatic reservoir (heavy metals and low $\mathrm{pH}$ ), near an ancient pyrite-cupric mine.

Several authors (Nebeker et al., 1984; Giesy and Hoke, 1989; Taylor et al., 1991; Ingersoll et al., 1995; Watts and Pascoe, 1996) recommend the Chironomus sp. 10-day growth test as a useful bioassay for evaluation of sediment toxicity, and standard protocols exist (e.g. ASTM, 2000; USEPA, 2000). Growth reduction in $C$. riparius larvae has been considered a sensitive response criterion (Taylor et al., 1991). In a study conducted by Giesy et al. (1988), growth reduction in $C$. tentans revealed to be as sensitive as the Microtox ${ }^{\circledR}$ 15-min EC50, and more discriminatory than the Microtox $^{\circledR}$ (15-min EC50) and Daphnia (48-h LC50) bioassays, using Detroit River sediments. A good agreement was verified between Hyalella azteca and $C$. tentans 10-day bioassays, using natural sediments (Becker et al., 1995). A correspondence between the larval growth reduction and the absence of indigenous chironomids in faunal surveys was also observed (Giesy et al., 1988). Furthermore, Sibley et al. (1997) found that changes in larval growth could be used to make valid predictions on reproductive and demographic parameters in C. tentans.

\section{Materials and methods}

\subsection{Test animals and culture conditions}

Midges (C. riparius) used in the experiments were obtained from laboratory cultures established at the Instituto do Ambiente e Vida, University of Coimbra. The culture unit was an enclosed transparent plastic box containing all the apparatus (beakers, etc.) necessary to complete the whole life cycle of the chironomids, and large enough to allow swarming and copulation of emerged adults (OECD, 2000). Cultures were maintained at $20 \pm 1{ }^{\circ} \mathrm{C}$, with a $14: 10 \mathrm{~h}$ light:dark cycle, which included a 90-min period of dawn and dusk. At the start of a new culture, approximately 30 first-instar larvae (3-4 days post-hatch) were introduced into glass beakers (11 cm in diameter) containing ASTM reconstituted hard water and acid washed, organic matter free (ignited for $8 \mathrm{~h}$ at $450{ }^{\circ} \mathrm{C}$ ) sea sand $(0.1-0.4 \mathrm{~mm}$ particle size range; supplied by Merck Co.). A suspension of ground Tetramin ${ }^{\circledR}$ (Tetrawerke, Germany) was then added as the single food source. Each beaker was gently aerated. Seven days later, larvae were either used in tests or transferred to new culture beakers with fresh media, food and sand (15 larva/beaker) until emergence occurred. Adults fed on a sucrose solution wetted paper, placed inside the culture unit. Fresh laid egg masses were transferred onto small plastic Petri dishes with culture medium for a period of 3-4 days, until eclosion occurred. The newborn larvae (1st instar) were then used to start a new culture. Testing, either in the field or laboratory, followed, with adaptations, the 10-day growth test standard protocols (Nebeker et al., 1984; ASTM, 2000; USEPA, 2000).

\subsection{In situ bioassay chamber design and general protocol}

Test-chambers were specially designed to carry out the in situ bioassays. The chambers consisted of a $1-\mathrm{m}$ long opaque PVC tube $(5.5 \mathrm{~cm}$ in diameter) with three sets of large lateral windows, covered with $200-\mu \mathrm{m}$ nylon mesh, and two openings, one at the top and the other at the bottom of the tube (Fig. 1A). The mesh was sealed to the windows with white thermal glue (supplied by Elis-Taiwan, Taiwan, ref. TN122/WS, with a chemical composition of $50 \%$ ethylene-vinyl-acetate copolymer, $45 \%$ synthetic hydrocarbon, and 5\% polyethylene wax), which has been shown to be non-toxic to cladocerans (Pereira et al., 1999). A three-piece chamber (Fig. 1B) was also developed since it could be more easily cleaned when recovering sediment and testorganisms at the end of the in situ bioassay. The three pieces were joined with two screw threads, which could

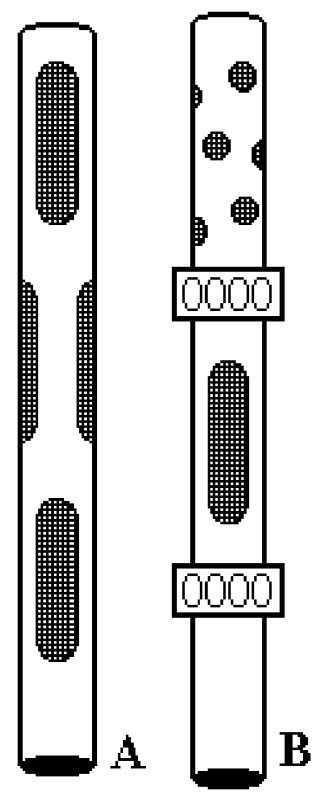

Fig. 1. In situ bioassay chambers: $\mathrm{A}$ - one-piece chamber (1-m long PVC tube with three sets of lateral openings covered with $200-\mu \mathrm{m}$ nylon mesh); B - three-piece chamber (each piece is joined to the other with a screw thread, for improved in situ recovery of test-organisms, Chironomus riparius). 
be unscrewed for better cleaning (one piece at a time). Previous chamber designs were attempted but recovery of organisms was typically poor. The final designs, presented here, aimed at maximum water-flow within the test-chamber, allowing acceptable dissolved oxygen levels inside the chamber (ideally, above $2.5 \mathrm{mg} / 1$, but acceptable up to $1.5 \mathrm{mg} / 1-$ ASTM, 2000; USEPA, 2000).

For the deployment of the chambers in the field, a $200-\mu \mathrm{m}$ mesh was placed at the bottom tip of the testchamber with the help of an elastic string (attached to a long line - retrieval string - which remained outside the chamber), and introduced into the water until it reached the ground. This procedure guaranteed the filtration of the water filling up the tube, avoiding entrance of possible indigenous predators. The mesh was then carefully removed by pulling the retrieval string. The test-chambers were then introduced into the sediment (approximately 10-20 cm deep, depending on the substrate type. The tops of the test-chambers were always above the waterline. When using artificial sediment (clean sand or a formulated sediment), a funnel and a thinner PVC tube (approximately $4 \mathrm{~cm}$ of diameter) allowed placing the sediment inside the chambers. Basically, the thinner tube was inserted into the chamber until it touched the ground, having its base covered with a $200-\mu \mathrm{m}$ mesh held by an elastic string. Afterwards, the artificial sediment was poured into the tube, through the funnel, and allowed to settle for a few minutes. The thinner tube was then gently removed while the mesh and the artificial sediment remained in the bottom, since the weight of the latter forced the release of the elastic string. This procedure allowed the placement of the artificial sediment after its settlement, reducing its dispersal to the water column and to the outside of the chambers, through the lateral windows.

Test-organisms, second to third instar (10 days posthatch) (Taylor et al., 1991), and food were added 1-2 days later through a specially designed apparatus (Fig. 2). Basically, this device consisted of a syringe (A) connected to a $1-\mathrm{m}$ piece of aquarium plastic tube (B), with a small weight $(\mathrm{C})$ in the opposite extremity. A small portion of filtered water (through a $200-\mu \mathrm{m}$ mesh) was sucked into the syringe and the chironomids transferred to the broader distal part ( $\sim 8 \mathrm{~cm}$ long; D) of the tube (maintained in a vertical position), by means of a plastic pipette. Pulling the piston gently lowered the water level in the tube/syringe system allowing the introduction of approximately $2 \mathrm{ml}$ of Tetramin ${ }^{\circledR}$ suspension. A $1 \mathrm{mg} /$ larva/day dose of Tetramin ${ }^{\circledR}$ was used in all experiments, as recommended as the optimal dose (Naylor and Rodrigues, 1995). The flexible tube was then inserted into the chamber and lowered until it reached the bottom. A small pressure at the base of the tube proved to be sufficient for preventing the early release of its content (food suspension and organisms)

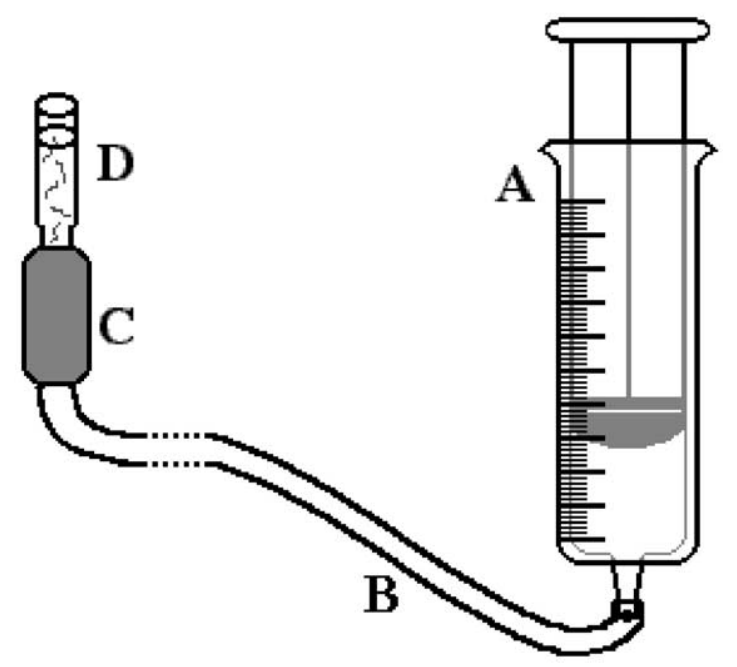

Fig. 2. Apparatus used for placing the chironomid larvae and food inside the test-chamber: A - syringe; B - plastic tube; C-small weight (sinker); D-broader distal part of the tube with test-organisms (Chironomus riparius).

while descending inside the test-chamber. Once near the bottom the syringe's piston was gently pushed, releasing the chironomids and food near the sediment surface, allowing them both to rapidly settle. This procedure guaranteed the settlement of the organisms in the sediment, and not along the vertical tube walls or in the lateral openings, as we had observed in preliminary experiments.

Chironomid larvae were transported to the field in small glass vials (approximately 20 larvae per $20-30 \mathrm{ml}$ of culture medium). At the end of the in situ bioassay, chambers were carefully withdrawn from the sediment, covering their base with one hand, in order to prevent the sediment core inside them from collapsing. The exterior of the chambers was always washed, since it could contain other organisms, including chironomids or predators. Only after this procedure was the sediment core released onto a plastic box. The chambers' interior was then thoroughly cleaned with a waterspout and inspected for organisms, especially the lateral windows. This procedure was performed in such a way that allowed the water used to clean the chambers to be collected in the plastic box, along with the sediment core. In the laboratory, sediment and water were sieved through a $500-\mu \mathrm{m}$ standard sieve and test organisms, as well as some indigenous organisms, were collected. Test C. riparius were killed in an acetone:water (1:3) mixture and length measurements of dead larvae were made, under a binocular stereoscope. Larvae were then placed in individual foil cups and dried for 48 hours at $60{ }^{\circ} \mathrm{C}$. Individual dry weights of dried larvae were obtained on a METTLER UMT2 microbalance to the nearest microgram. Recovery (recovered survivors), pupation and number of emerged adults (exuviae present) were 
registered. Recovery was used instead of survival because the possibility of some larvae being lost during the field retrieval could not be excluded.

\subsection{First experimental phase: in situ bioassay design optimization}

The developed bioassay chambers and protocols were primarily tested in a reference lagoon, due to its proximity to the laboratory. The main purpose of this initial phase was to evaluate the suitability of the developed chambers for exposing the organisms in situ, with acceptable recovery rates, and to choose the most adequate design. Bioassay chambers were hammered into the sediment, and organisms and food added the next day. Four treatments were performed, in order to optimize the in situ experimental design, as illustrated in Table 1. Both artificial (treatments A, B, and C) and natural (treatment D) sediment was used; the former was identical to the one used in laboratory cultures (i.e. clean sand). For treatments A, B and C, $120 \mathrm{~cm}^{3}$ of artificial sediment were added per chamber (it is the equivalent to a $5-\mathrm{cm}$ layer of sediment inside the chamber). The number of organisms per test-chamber was also tested ( 1 and 3 organisms per test-chamber), as well as the test-chamber type (see Fig. 1).

Test-chambers were checked every 3 days, being cleaned, if necessary, to remove accumulated particles from the mesh. Conductivity was measured with a WTW LF 92 conductivity meter, while measurement of $\mathrm{pH}$ was performed with a WTW $537 \mathrm{pH}$ meter. Dissolved oxygen concentrations (inside and outside the chambers) and temperature were determined with a WTW OXI 92 oxygen meter. Larvae were exposed for 12 days (instead of 10), since average field temperature was only $17{ }^{\circ} \mathrm{C}$. Simultaneously with the field treatments, two laboratory controls were used to assess the condition of the batch of larvae, one at $20^{\circ} \mathrm{C}$, another at $10{ }^{\circ} \mathrm{C}$.

\subsection{Second experimental phase: in situ toxicity testing}

In situ bioassay chambers were deployed at a contaminated site, located in a mine effluent. Besides these

Table 1

In situ bioassay design optimization (1st phase): type of sediment and chamber used, number of organisms per test-chamber (Org.), and number of test-chambers (Cham.) per in situ treatment

\begin{tabular}{lllcl}
\hline Treatment & Test-chamber & Org. & Cham. & Sediment type \\
\hline A & Whole-piece & 3 & 6 & Clean sand $^{\mathrm{a}}$ \\
B & 3-piece & 3 & 6 & Clean sand $^{\mathrm{a}}$ \\
C & Whole-piece & 1 & 18 & Clean sand $^{\mathrm{a}}$ \\
D & Whole-piece & 3 & 6 & Natural (local)
\end{tabular}

a Acid washed, organic matter free (ignited for $8 \mathrm{~h}$ at $450{ }^{\circ} \mathrm{C}$ ) sea sand (0.1-0.4 $\mathrm{mm}$ particle size range). chambers (treatment CNS), three additional in situ treatments were used: chambers with formulated sediment at the contaminated site (CAS), and two more treatments, a control (CTL) and a reference (REF), deployed at a reference site (in an upstream lagoon). Treatments consisted of four (CTL and REF) or three (CAS and CNS) whole-piece test-chambers, with five organisms per chamber. Formulated sediment, as described by Ribeiro et al. (1999) (consisting of $74 \%$ sand, $25 \%$ kaolinite clay and $1 \% \alpha$-cellulose), was used in CTL, REF and CAS treatments. Approximately 120 $\mathrm{cm}^{3}$ of formulated sediment (CTL, REF, and CAS) were added per chamber (equivalent to a 5 -cm layer of sediment inside the chamber). Contrarily to the previous experimental phase, the top of the tubes was covered with a piece of fine cloth to prevent entrance of local adult insects.

In the reference site, control test-chambers (CTL) were also used. Each control consisted of a normal whole-piece test-chamber placed inside a 20-1 plastic vessel containing ASTM hard water medium, with clean rocks and gravel keeping the chambers in a vertical position. The opening of the vessel was covered with a piece of fine mesh-like cloth to prevent oviposition by local adult insects during the test-period. The whole structure was then placed in the water, and fixated to the ground with nylon rope and rocks (in anchor-like structures) to avoid being dragged by the wind. Rocks and gravel inside the vessel also contributed to preventing the structure from floating.

When using the formulated sediment (CTL, REF and CAS) and before deploying the chamber, a small depression was previously made in the sediment to allow the direct contact between the formulated sediment inside the chamber and the surrounding natural sediment and pore-water (Fig. 3). Furthermore, also when using the formulated sediment, a $200-\mu \mathrm{m}$ mesh was glued to the base of the chambers, covering it, in

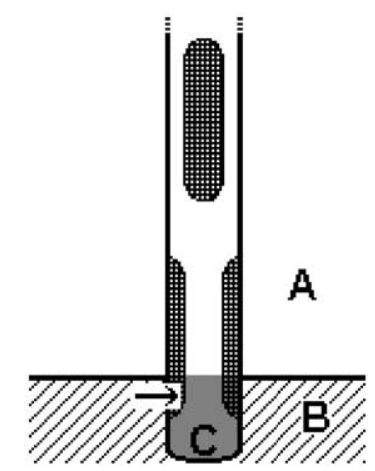

Fig. 3. In situ bioassay chamber after deployment in the field. Chamber is held vertically along the water column (A). When natural sediment is used, chambers are hammered into the substrate (B). when formulated sediment (C) is used, the chamber is placed in a previously made depression. Arrow illustrates the entrance of the surrounding pore water and fine $(<200 \mu \mathrm{m})$ particles. 
order to prevent the entrance of large indigenous organisms. Physical and chemical parameters $(\mathrm{pH}$, temperature and dissolved oxygen) were monitored at the beginning and end of the experiment, as described for the first experimental phase. Larvae were exposed for 7 days (instead of 10), since average temperature was $25{ }^{\circ} \mathrm{C}$. The shortening of the duration of the bioassay aimed at avoiding high pupation and emergence, since high growth rates were to be expected at these temperatures. A laboratory control (with formulated sediment) was also performed (at $20{ }^{\circ} \mathrm{C}$ ) during the test period, using the same batch of larvae.

\subsection{Laboratory bioassay}

A week after the end of the in situ bioassay, a laboratory bioassay was performed using natural sediment and water collected from the reference and contaminated sites. Composite sediment samples were collected with a PVC tube, by scraping the upper layer (2-5 cm, as recommended by Hill et al., 1993) of the sediment. These samples were kept in airtight plastic containers, and placed in the dark. Water samples were collected near the surface with 1 or 5-1 plastic bottles. Both water and sediment samples were transported and stored in the dark at $4{ }^{\circ} \mathrm{C}$ until the beginning of the test. In the laboratory, sediment samples were visually searched and visible indigenous animals and large debris (leaves, etc.) removed with forceps. Small subsamples were taken for analysis of organic matter content (percent volatile solids) and particle size distribution (using a standard sieving technique-Buchanan and Kain, 1971). Organic matter content was determined as the percentage of initial dry weight of sample remaining after igniting in a muffle furnace at $450{ }^{\circ} \mathrm{C}$ for $6 \mathrm{~h}$ (adapted from Buchanan and Kain, 1971).

Whole-sediment laboratory bioassays with C. riparius followed, with some modifications, the standard 10-day larval growth test (ASTM, 2000; Taylor et al., 1991; Ingersoll et al., 1995; Nebeker et al., 1984). All major SETAC recommendations towards bioassay experimental design (test vessel, sediment and overlying water) were followed (Hill et al., 1993). Three treatments were used: a control (CTL) consisting of ASTM hard water and formulated sediment (as described by Ribeiro et al., 1999), while REF and CONT utilized water and sediment from the reference and contaminated site, respectively. Four replicate test vessels $(250 \mathrm{ml}$ glass flasks, with $5.5 \mathrm{~cm}$ in diameter), with five organisms each, were used in all treatments, as performed in the in situ bioassay. Sediment and overlying water were added the day before starting the test. A 2-cm layer of sediment was carefully placed at the bottom of the beakers. Water was added without disturbing the sediment (using a plastic object to refrain water thrust), up to 8 -cm depth yielding a sediment:overlying water depth ratio of 1:4, as recom- mended by OECD (2000). After a 24-h conditioning period, gentle aeration was started and organisms added (day 0) $30 \mathrm{~min}$ later. While adding the chironomids, aeration was stopped for a 30-min period, allowing larvae to settle properly. Food (ground Tetramin ${ }^{\mathbb{R}}$ ) was added in a single $1 \mathrm{mg} /$ larva/day dose at day 0 .

At day 10, organisms were collected (with a $500-\mu \mathrm{m}$ sieve) and killed with acetone:water (1:3). Body length and dry weight were estimated, as described before for the in situ bioassays. Mortality, pupation and number of emerged adults were also determined at the end of the test. Physical and chemical parameters $(\mathrm{pH}$, temperature, dissolved oxygen, and conductivity) were measured on days 0 and 10 of the experiment.

\subsection{Study sites}

The first phase of this study was conducted at a reference site (Braças Lagoon), where no contamination sources are known. The aim of this initial work was to optimize the bioassay chambers and procedures (see earlier). São Domingos mine, located in the Southeast of Portugal, was the location chosen for the second experimental phase, where the previously optimized in situ chambers and protocol were used to compare laboratory and in situ results. The history and topography of this abandoned pyrite-cupric mine are described elsewhere; Lopes et al., 1999a,b; Pereira et al., 1999, 2000). Reference lagoons and a contaminated mine effluent coexist in the area, and no other significant contamination sources are known (e.g. pesticides, industrial discharges or urban runoffs). Pyrite oxidation is still occurring in the mine tailings, producing acid mine drainage, with low $\mathrm{pH}$ and very high concentrations of metals (in decreasing order: $\mathrm{Fe}, \mathrm{Al}, \mathrm{Zn}, \mathrm{Cu}, \mathrm{Cd}, \mathrm{Cr}, \mathrm{Pb}$, As; Lopes et al., 1999a,b; Pereira et al., 2000), which enters the reservoir of the Chança River dam (see figure in Pereira et al., 1999).

\subsection{Statistical analysis}

Larval dry weights and body lengths were analyzed for differences with one-way analysis of variance (ANOVA), followed by Tukey HSD multiple comparison test, when applicable. Pupation, emergence and mortality/recovery were analyzed for significance using the Fisher exact test.

\section{Results}

\subsection{First experimental phase: in situ bioassay optimization}

The first phase of the study was conducted at Braças Lagoon in the end of March. Relatively low temperatures (ranging from 16.4 to $18{ }^{\circ} \mathrm{C}$, during the day) were registered during the course of the experiment. 
Conductivity, oxygen and $\mathrm{pH}$ values were stable during the 12 days exposure, with mean values of $325 \mu \mathrm{S} / \mathrm{cm}$, $9.0 \mathrm{mg} / \mathrm{l}$ and 7.5, respectively. Oxygen levels within the chambers were acceptable, with the lowest value being $83 \%$ of saturation. Laboratory controls (at 10 and $20{ }^{\circ} \mathrm{C}$ ) demonstrated the fitness of the batch of larvae used. At $20^{\circ} \mathrm{C}$ all the larvae pupated and two of them emerged, while at $10{ }^{\circ} \mathrm{C}$ larvae $(n=10)$ attained a length of $10.17 \pm 1.18 \mathrm{~mm}$ and a dry weight of $665 \pm 213 \mu \mathrm{g}$.

Recovery of the organisms was generally low (25$58.3 \%$ ) (Table 2). The lowest (although not significant) recovery was recorded in $\mathrm{C}(25 \%)$, where only one larva was used, and in D (28\%), where natural sediment was used. The recovery rates of A $(55.6 \%)$ and $\mathrm{B}(58.3 \%)$ were very similar, suggesting similar performance of whole-piece and three-piece chambers in recovering testorganisms. Analysis of body lengths and dry weights found no significant differences between treatments (Table 2). Along with test-organisms, indigenous animals were also recovered from the tested sediments, especially in D. The most frequently found organisms were other chironomid species (other than C. riparius) and some Ephemeroptera nymphs (namely Caenis sp.).

\subsection{Second experimental phase: in situ toxicity testing}

The in situ bioassay was carried out in the beginning of June in S. Domingos Mine, with temperature ranging between 23 and $27.5{ }^{\circ} \mathrm{C}$ (Table 3). Oxygen concentration was near saturation in both stations, and $\mathrm{pH}$ values were slightly alkaline in the reference site and neutral in the contaminated site (Table 3). Sediment from the reference site had less than half the organic matter content of the sediment from the contaminated site (Table 4). Both sediments were also very different in terms of particle size distribution (Table 4). Sediment from the contaminated site was almost exclusively comprised of silt and clay $(0.063 \mathrm{~mm})$, while sediment from the reference site was mainly sand (fine and medium sand: $0.063-0.1 \mathrm{~mm}$ ), although it also had a large percentage of finer particles (approximately 30\%).

Table 2

In situ bioassay design optimization (1st phase): number of larvae per treatment $(\mathrm{N})$, recovery rates (Recov.), body length (average \pm S.D., in $\mathrm{mm}$, and sample size in brackets) and dry weight (average \pm S.D., in $\mu \mathrm{g}$, and sample size in brackets) of Chironomus riparius larvae after 12day exposure to the four in situ treatments

\begin{tabular}{lllcl}
\hline Treatment & $\mathrm{N}$ & Recov. $(\%)$ & Length $(\mathrm{mm})$ & Dry weight $(\mu \mathrm{g})$ \\
\hline $\mathrm{A}$ & 18 & 55.6 & $9.86 \pm 1.00(10)$ & $486 \pm 179(9)$ \\
$\mathrm{B}$ & $12^{\mathrm{a}}$ & 58.3 & $10.26 \pm 1.07(7)$ & $478 \pm 193(7)$ \\
$\mathrm{C}$ & $16^{\mathrm{a}}$ & 25.0 & $9.35 \pm 1.01(4)$ & $336 \pm 103(4)$ \\
$\mathrm{D}$ & 18 & 27.8 & $9.27 \pm 1.12(5)$ & $321 \pm 86(5)$ \\
\hline
\end{tabular}

a Two of the chambers were rejected due to loss of sediment or damage.
Recovery rates in the in situ bioassay (Table 5) ranged from $73.3 \%$ to $100 \%$, being significantly lower in the treatment with natural sediment at the contaminated site (CNS) than in the in situ control (CTL). Comparatively to CTL (Table 5), the reduction on pupation was significant at the reference site (REF: $P<0.05)$, at the contaminated site using natural sediment (CNS: $P<0.01)$, and at the contaminated site using formulated sediment (CAS: $P<0.001)$. Emergence (Table 5) was significantly reduced in all treatments (REF, CAS and CNS) when compared to that of the CTL. Only in CAS, larval body length and dry weight (Table 5) were significantly lower than the in situ control (CTL) (one-way ANOVAs, $\quad F_{(3,26)}=21.0, \quad P<10^{-6}$ and $F_{(3,26)}=44.8$, $P<10^{-9}$, respectively). A laboratory control (at $20^{\circ} \mathrm{C}$ ) used the same batch of larvae $(n=17)$, which attained a length of $11.76 \pm 1.11 \mathrm{~mm}$ and a dry weight of $1510 \pm 378$ $\mu \mathrm{g}$, which demonstrated the fitness of this batch. Pupation $(20 \%)$ also occurred in this laboratory control.

\subsection{Laboratory bioassay}

Physical and chemical parameters were almost constant during the laboratory bioassay, carried out a week after the end of the in situ bioassay, and a slight increase in $\mathrm{pH}$ of the contaminated sediment sample, comparing to in situ values, was recorded (Table 3). The laboratory bioassay was not able to discriminate toxicity of the

Table 3

Range of physical and chemical parameters registered in the in situ and laboratory bioassays

\begin{tabular}{|c|c|c|c|c|}
\hline \multirow[t]{2}{*}{ Parameters } & \multicolumn{2}{|c|}{ In situ bioassay } & \multicolumn{2}{|c|}{ Laboratory bioassay } \\
\hline & Reference & Contaminated & Reference & Contaminated \\
\hline Temp. $\left({ }^{\circ} \mathrm{C}\right)$ & $24-25$ & $23-27.5$ & 20 & 20 \\
\hline D.O. $(\mathrm{mg} / \mathrm{l})$ & $9.3-9.9$ & $8.3-8.7$ & $8.7-8.9$ & $7.5-7.7$ \\
\hline $\mathrm{pH}$ & $8.0-8.7$ & $6.8-7.2$ & $7.1-7.8$ & $7.4-7.7$ \\
\hline Cond. $(\mu \mathrm{s} / \mathrm{cm})$ & $-\mathrm{a}^{\mathrm{a}} \mathrm{c}$ & $-\mathrm{a}^{\mathrm{a}} \mathrm{r}$ & $260-270$ & $230-240$ \\
\hline
\end{tabular}

a No values available.

Table 4

Particle size distribution and organic matter content (volatile solids) of the tested sediments [organic matter content is represented with associated S.D. $(n=4)]$

\begin{tabular}{lcc}
\hline Parameters & Reference & Contaminated \\
\hline Particle size $(\mu \mathrm{m})$ & & \\
$>2000 \mu \mathrm{m}$ & 12.60 & 0.00 \\
$1000-2000 \mu \mathrm{m}$ & 8.88 & 0.03 \\
$500-1000 \mu \mathrm{m}$ & 8.66 & 0.02 \\
$250-500 \mu \mathrm{m}$ & 11.11 & 0.08 \\
$125-250 \mu \mathrm{m}$ & 15.84 & 0.15 \\
$63-125 \mu \mathrm{m}$ & 13.13 & 1.35 \\
$<63 \mu \mathrm{m}$ & 29.90 & 98.42 \\
& & $5.79 \pm 0.15$ \\
\hline
\end{tabular}


Table 5

Body length (average $\pm \mathrm{SD}$, in $\mathrm{mm}$, and sample size in brackets), dry weight (average \pm S.D., in $\mu \mathrm{g}$, and sample size in brackets) and recovery, pupation and emergence rates of Chironomus riparius larvae at the end of the in situ bioassay

\begin{tabular}{|c|c|c|c|c|c|}
\hline Treatment & Body length (mm) & Dry weight $(\mu \mathrm{g})$ & Recovery (\%) & Pupation $(\%)$ & Emergence $(\%)$ \\
\hline CTL & $11.92 \pm 1.77(3) \mathrm{a}$ & $693 \pm 31(3) a, b$ & 100.0 & 80.0 & 35.0 \\
\hline REF & $11.76 \pm 1.11(7) \mathrm{a}$ & $633 \pm 111(7) a$ & 80.0 & $33.3^{*}$ & $0.0^{*}$ \\
\hline CAS & $8.68 \pm 0.56(11) b$ & $258 \pm 36(11) \mathrm{c}$ & 100.0 & $0.0 * * *$ & $0.0^{*}$ \\
\hline CNS & $12.06 \pm 1.31(9) \mathrm{a}$ & $836 \pm 179(9) b$ & $73.3^{*}$ & $18.2 * *$ & $0.0^{*}$ \\
\hline
\end{tabular}

ANOVA (Body length): $F_{(3,26)}=21.0, P<10^{-6}$; ANOVA (Dry weight): $\mathrm{F}_{(3,26)}=44.8, P<10^{-9}$. Different letters $(\mathrm{a}, \mathrm{b}, \mathrm{c})$ represent significant differences between treatments $(P \leqslant 0.05)$. $* P \leqslant 0.05$. $* * P \leqslant 0.01 . * * * P \leqslant 0.001$.

Table 6

Body length (average $\pm \mathrm{SD}$, in $\mathrm{mm}$, and sample size in brackets), dry weight (average \pm S.D., in $\mu \mathrm{g}$, and sample size in brackets) and survival, pupation and emergence rates of Chironomus riparius larvae at the end of the laboratory bioassay

\begin{tabular}{|c|c|c|c|c|c|}
\hline Treatment & Body length (mm) & Dry weight $(\mu \mathrm{g})$ & Survival (\%) & Pupation (\%) & Emergence $(\%)$ \\
\hline CTL & $12.51 \pm 1.09$ & $1308 \pm 581$ & $90.0 \%$ & $72.2 \%$ & $5.6 \%$ \\
\hline REF & $12.38 \pm 1.48(5)$ & $1200 \pm 385(5)$ & $90.0 \%$ & $72.2 \%$ & $11.1 \%$ \\
\hline CONT & $12.65 \pm 1.59(8)$ & $1274 \pm 453(7)$ & $75.0 \%$ & $46.7 \%$ & $6.7 \%$ \\
\hline
\end{tabular}

tested sediments, since no significant differences were found between treatments in any of the measured endpoints (Table 6). Although non-significant, an apparent reduction in survival and in pupation was observed in the sediment from the contaminated site (CONT).

\section{Discussion}

\subsection{In situ bioassay experimental design}

In general terms, the described in situ bioassay protocol and chambers were suitable for performing in situ sediment toxicity bioassays. The developed chamber utilized similar materials to the ones described by Pereira et al. (1999), with the exception of the polypropylene beaker, which was replaced by the PVC tube. The choice for the materials used in this study (PVC tube and nylon mesh) implies additional care when performing in situ bioassays at contaminated sites, since adsorption of several compounds (especially organic) must be accounted for. For this reason, a period between the deployment of the chambers and the introduction of food and organisms should always be provided, as performed in the present study (24-48 h).

Top caps, which were added to the later test-chamber design, were designed to prevent the entrance of flying indigenous organisms (occasionally, adult chironomids and culicids were found in the aerial part of the bioassay chamber during preliminary trials, when the mesh was not present). Base caps in the tubes were used in the treatments with formulated sediment to prevent the entrance of benthic indigenous organisms. The choice for whole-piece chambers for the later test-chamber design was based on cost-effectiveness, since their recovery rates were similar to the ones recorded in the more expensive three-piece chambers. Ribeiro et al. (1999) developed a formulated sediment that had a better performance (helping chironomids in tube building) than sand, which dictated the replacement of sand (artificial sediment used in the first experimental phase) by this formulated sediment, in the second experimental phase. This change aimed at lowering mortality and enhancing growth performance. Recovery rates in the second experimental phase were higher (close to $100 \%$ ) than those in the first experimental phase, and indigenous animals were less frequently found in the test sediments.

The use of artificial sediments in bioassays has been limited almost exclusively to laboratorial use, as an attempt to standardize sediment bioassays and to reduce the influence of possible confounding factors in toxicity assessment, such as sediment organic carbon content and grain size (Hill et al., 1993; Naylor and Rodrigues, 1995; Ribeiro et al., 1999). Ristola et al. (1999) and Ankley et al. (1994) have shown that benthic invertebrates, including Chironomus spp., may respond to sediment characteristics, which can lead to biased conclusions on sediment toxicity. The incorporation of formulated sediment in the in situ bioassay, supplementing natural sediment testing, aimed at minimizing the natural variability due to physical and chemical differences among sediments or due to interactions with indigenous organisms. On one hand, this was successfully accomplished as noticed by the consistent high recovery of organisms in artificial sediments. Furthermore, formulated sediments fulfill the need for reproducibility and standardization (Hill et al., 1993). On the other hand, two fundamental problems arise from the use of formulated sediments in situ: (1) they do not 
represent a realistic scenario, being counterproductive with the environmental realism offered by the in situ exposure; (2) it is likely that organisms respond differently between formulated and natural sediments, as observed in the in situ bioassay performed in the mine effluent. Furthermore, when using the formulated sediment, the experimental in situ protocol involves sediment disturbance. This appears to be the main weakness of using artificial sediments, since physical and chemical alterations of the substrate occur, and hence changes in contaminant bioavailability (Burton, 1991). Allowing some time between test-chamber deployment and the introduction of organisms will allow the system to reach equilibrium, but the duration of this time interval is unknown. Therefore, artificial sediments (CAS, REF, and CTL, in the present study) should only be used in in situ testing to supplement the information obtained with natural sediments (CNS), and not to replace the use of natural sediments.

Other authors have developed in situ bioassay chambers and protocols for in situ toxicity testing with benthic invertebrates. DeWitt et al. (1999) described a simple bioassay with amphipods, placed in cages in intertidal sediments. The need for tidal exposure during cage deployment and retrieval restricts the generalized application of such a bioassay. A similar chamber had already been proposed by Chappie and Burton (1997) to perform in situ bioassays with $C$. tentans and Hyalella azteca, which was recently applied in an actual field situation (Tucker and Burton, 1999). In these bioassays, only artificial sediment (or no sediment whatsoever) was used and only survival was monitored. Organisms were introduced in the test chambers either inside their tubes (Chappie and Burton, 1997) or already in the sediment (Tucker and Burton, 1999). Tube building has been considered to be of vital importance to many chironomid larvae (Naylor and Rodrigues, 1995); because contaminants could affect tube building behavior, it seems preferable to add organisms without their tube. More recently, Sibley et al. (1999) developed an in situ bioassay chamber to use with benthic invertebrates, including $C$. tentans (growth and survival). The complexity of the bioassay chamber developed by Sibley et al. (1999) and its cost (approximately 28\$ US), are a major drawback. These authors suggest that chironomids should be added inside their case, which has been previously discussed. Releasing the larvae near the top of the test-chamber, instead of doing so near the substrate is somewhat problematic, since we observed, in preliminary designs we tested, that larvae could fixate and build a new case in the interior walls of the testchamber (especially near the water exchange ports). This could lead to accounting these organisms as dead, if not retrieved. If these organisms were in fact retrieved at the end of the bioassay, they would have been exposed exclusively to water-column contaminants.
The performance of the in situ bioassay chamber and protocol described here is, at least, comparable to that of other existing designs. Its main advantage is its simplicity and low cost (roughly, the design of the wholepiece test-chamber had an estimated cost of less than $8 \$$ US). 10-day exposures have been successfully conducted using the described procedures, in both natural and artificial sediments, and its use can be broadened to other taxa, including indigenous species. Amphipods, oligochaetes, bivalves, and insect larvae (e.g. Ephemeroptera, Trichoptera) have also shown to be suitable organisms for in situ sediment toxicity testing (Crane et al., 1995; Monson et al., 1995; Shaw and Manning, 1996; Chappie and Burton, 1997; DeWitt et al., 1999; Sibley et al., 1999; Schulz and Liess, 1999; Tucker and Burton, 1999; Soucek et al., 2000).

\subsection{Confounding factors}

Low temperatures may have contributed to the low recoveries observed in the first experimental phase. Other authors have observed low survival of $C$. tentans larvae at field temperatures ranging from 10 to $15{ }^{\circ} \mathrm{C}$ (Chappie and Burton, 1997; Sibley et al., 1999). The subject of temperature-related effects on in situ survival of benthic invertebrates is further discussed by Chappie and Burton (1997). Water-flow within the test-chambers was sufficient to maintain high oxygen levels inside testchambers, complying with the standard bioassay requirements (preferably higher than $2.5 \mathrm{mg} / 1$, never below $1.5 \mathrm{mg} / \mathrm{l}$ ) (USEPA, 2000; ASTM, 2000). Light penetration is also an aspect to take into account in the test-chamber design. Ideally, test-chambers should be made of transparent material, but this did not appear to be a problem to $C$. riparius in this study. However, since light intensity was not measured inside the test-chambers and its effects were not tested, it is not possible to accurately assess the influence of this confounding factor. Still, when dealing with photoactivated contaminants, light intensity inside and outside the chambers should be measured, since this could influence the responses obtained (Monson et al., 1995; Ireland et al., 1996).

The presence of indigenous organisms was registered in the present bioassay, especially when natural sediments were tested. Indigenous organisms pose two types of difficulties in in situ sediment toxicity assessment (Chappie and Burton, 1997): (1) local fauna may interact (as competitors or predators) with test-organisms, biasing toxicity interpretation; (2) difficulties in discriminating test-species from naturally occurring species may be posed. The latter factor was not a problem in the present study, since it was possible to easily distinguish $C$. riparius from local chironomids. However, marking the test-organisms may be important when deploying test-chambers where natural populations exist, thus solving misidentification problems (Crane et 
al., 2000). Indigenous fauna has been shown to affect growth (Reynoldson et al., 1994) and survival (Sibley et al., 1999; Crane et al., 2000) of Chironomus spp..

The mesh size used in the present study $(200 \mu \mathrm{m})$ is similar to the one used by Sibley et al. (1999) and allowed satisfactory aeration, whilst retaining test organisms and blocking the entrance to large indigenous organisms. Even so, some chironomid larvae and other small invertebrates could enter the test-chamber. Although other authors (Chappie and Burton, 1997; Pereira et al., 1999) have reduced the mesh size (from 149 to $74 \mu \mathrm{m}$, and from 150 to $50 \mu \mathrm{m}$, respectively) of the in situ test-chambers to prevent entrance of indigenous fauna, it seems preferable not to do it in this case in order not to compromise proper water flow within the test-chambers.

In the second experimental phase, pupae and adult chironomids (or exuviae) were found at the end of the bioassay. Although many authors (e.g. Watts and Pascoe, 1996; Benoit et al., 1997) recommend assessing emergence and pupation along with growth and survival of chironomids, this type of approach should be avoided with the described in situ experimental design. The assessment of reproductive endpoints would require an additional number of replicate test-chambers, with more test-organisms, and a proper adult (imago) collection method. Starting the in situ bioassay with younger larvae (6-7 days post-hatch instead of 10) appears to be a good solution, although younger larvae are probably more susceptible to handling stress or temperature fluctuations. In fact, Nebeker et al. (1984) first suggested the use of older (3rd instar) larvae in in situ bioassays, instead of the 2 nd instar, to prevent such mortality. However, it seems preferable to use younger larvae, than to lose statistical power (less organisms available to quantify growth) and to increase the bias of accounting for emerged adults. Nevertheless, chironomid growth rates at different temperatures should be determined before deploying the in situ bioassay, in order to predict the duration of the bioassay, which would allow the chironomids to reach the 4 th instar or pupae without emerging.

\subsection{Laboratory versus in situ bioassay}

While no lethal or sublethal toxicity was detected in the laboratory, differences were found between the contaminated site and the in situ control, in the in situ bioassay. First, a lower recovery was found in the treatment with natural sediment at the contaminated site (CNS). Second, a significant reduction in pupation $(0-18.2 \%)$ was recorded at the contaminated site using both types of sediment (CAS and CNS, respectively). Heavy metals present in the sediment appear, thus, to be responsible for a delay in the larval development of $C$. riparius, which has been previously described for other metal-contaminated sediments (Wentsel et al., 1978; Watts and Pascoe, 1996). A less pronounced developmental delay was also found at the reference site in the in situ bioassay, using formulated sediment (REF), which could be due to a higher water quality (ASTM culture medium) used in the in situ control (CTL).

Third, a significant growth inhibition was found at the contaminated site using formulated sediment (CAS). However, growth was not significantly depressed when using natural sediment in situ (CNS). The relatively high organic matter content of this natural sediment may have contributed to this discrepancy between natural and formulated sediment. Organic matter present in the sediments may have enhanced chironomid growth at this site, while at the same time reducing contaminant bioavailability, as found by Stuijfzand et al. (2000), which might explain the absence of toxicity where natural sediment was used (CNS). Stuijfzand et al. (2000) had similar results in an in situ experiment, suggesting that tolerance to pollution in C. riparius was an outcome of its ability to profit from organic enrichment. Thus, the significant reduction of growth and pupation registered in CAS could be related with the absence of a compensatory effect of organic matter (Stuijfzand et al., 2000), observed in the natural sediments but not in the organic-matter-free formulated sediment. In fact, growth of chironomids appeared to be slightly stimulated in the organic-enriched natural sediments (CNS). Watts and Pascoe (1996) also observed differences between formulated and natural sediments, with significant growth reduction in $C$. riparius with copperspiked formulated sediment and absence of toxicity with copper-spiked natural sediment. These authors briefly focused on sediment/contaminant complexing and bioavailability as a possible explanation.

Standard laboratory toxicity testing did not detect any significant alterations in any of the endpoints assessed, but toxic effects were detected in the field (see earlier). Since formulated sediment apparently overestimated toxicity, it seems adequate to compare the contaminated laboratory treatment (CONT) only with the in situ treatment in the contaminated natural sediment (CNS). Pupation was the only endpoint affected by the contaminated sediment, and this was significant only in situ. Differences between laboratory and field can be due to two main reasons, or both: (1) fluctuating environmental conditions in the field enhanced the toxic effect of the contaminated sediment; (2) toxicity of the sediment was reduced during transportation and storage, which is not infrequent when dealing with sediments, especially if the contamination level is low (Beiras et al., 1998), which seems to be the case in the current study. The slight differences in $\mathrm{pH}$ registered between field and laboratory could be a consequence of transportation and storage of water samples and sediment. 


\section{Conclusions}

The developed test-chamber and protocol were suitable for exposing and retrieving $C$. riparius in situ, in a cost-effective and simple way. It presents some advantages relatively to other similar experimental designs for in situ sediment toxicity testing, namely the simplicity of the test-chamber and of the organism-release mechanism. The presented test-chamber design is mainly applicable to low-energy, shallow systems. For a better simulation of natural conditions, chambers should be made of transparent material instead of the opaque PVC. The supplementary use of artificial sediments is useful as a means of reducing potentially confounding natural variability, thus contributing to a sounder interpretation of results with natural sediments.

In situ toxicity was not detected in the laboratory bioassay, suggesting that laboratory-to-field extrapolation can sometimes be biased. This type of comparisons should be broadened to other bioassays, comprising several sites with different contamination sources, in order to assess the ecological significance of standard laboratory bioassays. In fact, we think that laboratory testing should preferably be complemented with in situ bioassays, particularly if site-specific ecotoxicological information is needed.

\section{Acknowledgements}

B.B. Castro was a recipient of a M.Sc. grant from Fundação para a Ciência e a Tecnologia (Portugal)PRAXIS XXI. This work was partially funded by Fundação para a Ciência e a Tecnologia (project SENSOR, ref. PRAXIS/PCNA/C/BIA/0157/96) and by the European Commission (project TROCA-WET, ref. ERBIC18-CT98-0264). Authors wish to thank O. Sobral and P. Raposo for their contributions to the development of previous chamber designs, and to I. Lopes for assistance during fieldwork.

\section{References}

Ankley, G.T., Benoit, D.A., Balogh, J.C., Reynoldson, T., Day, K.E., Hoke, R.A., 1994. Evaluation of potential confounding factors in sediment toxicity tests with three freshwater benthic invertebrates. Environ. Toxicol. Chem. 13 (4), 627-635.

ASTM, 2000. Test method for measuring the toxicity of sedimentassociated contaminants with freshwater invertebrates. E 1706-00. In: Annual Book of American Society for Testing and Materials Standards, ASTM, Philadelphia, USA.

Becker, D.S., Rose, C.D., Bigham, G.N., 1995. Comparison of the 10 days freshwater sediment toxicity tests using Hyalella azteca and Chironomus tentans. Environ. Toxicol. Chem. 14, 2089-2094.

Beiras, R., His, E., Seaman, M.N.L., 1998. Effects of storage temperature and duration on toxicity of sediments assessed by Crassos- trea gigas oyster embryo bioassay. Environ. Toxicol. Chem. 17, 2100-2105.

Benoit, D.A., Sibley, P.K., Juenemann, J.L., Ankley, G.T., 1997. Chironomus tentans life cycle test: design and evaluation for use in assessing toxicity of contaminated sediments. Environ. Toxicol. Chem. 16, 1165-1176.

Buchanan, J.B., Kain, J.M., 1971. Measurement of the physical and chemical environment. In: Holme, N.A., McIntyre, A.D. (Eds.), Methods for the Study of Marine Benthos-IBP Handbook $n^{\circ} 16$. Blackwell Scientific Publications, UK, pp. 30-58.

Burton, G.A., 1991. Assessing the toxicity of freshwater sediments. Environ. Toxicol. Chem. 10, 1585-1627.

Chappie, D.J., Burton, G.A., 1997. Optimization of in situ bioassays with Hyalella azteca and Chironomus tentans. Environ. Toxicol. Chem. 16, 559-564.

Crane, M., Delaney, P., Mainstone, C., Clarke, S., 1995. Measurement by in situ bioassay of water quality in an agricultural catchment. Water Res. 29, 2441-2448.

Crane, M., Higman, M., Olsen, T., Simpson, P., Callaghan, A., Fisher, T., Kheir, R., 2000. An in situ system for exposing aquatic invertebrates to contaminated sediments. Environ. Toxicol. Chem. 19 (11) 2715-2719.

DeWitt, T.H., Hickey, C.W., Morrisey, D.J., Nipper, M.G., Roper, D.S., Williamson, R.B., Van Dam, L., Williams, E.K., 1999. Do amphipods have the same concentration-response to contaminated sediment in situ as in vitro? Environ. Toxicol. Chem. 18, 1026-1037.

Giesy, J.P., Graneym, R.L., Newsted, J.L., Rosiu, C.J., Benda, A., 1988. Comparison of three sediment bioassay methods using Detroit River sediments. Environ. Toxicol. Chem. 7, 483-498.

Giesy, J.P., Hoke, R.A., 1989. Freshwater sediment toxicity bioassessment: rationale for species selection and test design. J. Great Lakes Res. 15, 539-569.

Hill, I.R., Mathiessen, P., Heimbach, F. (Eds.), 1993. Guidance Document on Sediment Toxicity Tests and Bioassays for Freshwater and Marine Environments. SETAC-Europe, Brussels, Belgium.

Ingersoll, C.G., Ankley, G.T., Benoit, D.A., Brunson, E.L., Burton, G.A., Dwyer, F.J., Hoke, R.A., Landrum, P.F., Nordberg-King, T.J., Winger, P.V., 1995. Toxicity and bioaccumulation of sediment-associated contaminants using freshwater invertebrates: a review of methods and applications. Environ. Toxicol. Chem. 14, 1885-1894.

Ireland, D.S., Burton, G.A., Hess, G.G., 1996. In situ toxicity evaluations of turbidity and photoinduction of polycyclic aromatic hydrocarbons. Environ. Toxicol. Chem. 15, 574-581.

Lopes, I., Gonçalves, F., Soares, A.M.V.M., Ribeiro, R., 1999a. Discriminating the ecotoxicity due to metals and to low $\mathrm{pH}$ in acid mine drainage. Ecotoxicol. Environ. Saf 44, 207-214.

Lopes, I., Gonçalves, F., Soares, A.M.V.M., Ribeiro, R., 1999b. Ecotoxicological tools in the remediation of acid mine drainage. Toxicol. Environ. Chem. 70, 441-460.

Monson, P.D., Ankley, G.T., Kosian, P.A., 1995. Phototoxic response of Lumbriculus variegatus to sediments contaminated by polycyclic aromatic hydrocarbons. Environ. Toxicol. Chem. 14, 891-894.

Naylor, C., Rodrigues, C., 1995. Development of a test method for Chironomus riparius using a formulated sediment. Chemosphere 31 , 3291-3303.

Nebeker, A.V., Cairns, M.A., Gakstatter, J.H., Malueg, K.W., Schuytema, G.S., Krawczyk, D.F., 1984. Biological methods for determining toxicity of contaminated freshwater sediments to invertebrates. Environ. Toxicol. Chem. 3, 617-630.

OECD, 2000. Sediment-water chironomid toxicity test using spiked water-draft document. OECD guidelines for the testing of chemicals - proposal for a new guideline 219.

Pereira, A.M.M., Soares, A.M.V.M., Gonçalves, F., Ribeiro, R., 1999. Test-chambers and test procedures for in situ toxicity testing with zooplankton. Environ. Toxicol. Chem. 18, 1956-1964. 
Pereira, A.M.M., Soares, A.M.V.M., Gonçalves, F., Ribeiro, R., 2000. Water-column, sediment and in situ chronic bioassays with cladocerans. Ecotoxicol. Environ. Saf. 47, 27-38.

Reynoldson, T.B., Day, K.E., Clarke, C., Milani, D., 1994. Effect of indigenous animals on chronic end points in freshwater sediment toxicity tests. Environ. Toxicol. Chem. 13, 973-977.

Ribeiro, R., Kelly, L.A., Gonçalves, F., Burton, G.A., Soares, A.M.V.M., 1999. New artificial sediment for Chironomus riparius toxicity testing. Bull. Environ. Contam. Toxicol. 63, 691-697.

Ristola, T., Pellinen, J., Ruokolainen, M., Kostamo, A., Kukkonen, J.V.K., 1999. Effect of sediment type, feeding level, and larval density on growth and development of a midge (Chironomus riparius). Environ. Toxicol. Chem. 18 (4), 756-764.

Schulz, R., Liess, M., 1999. Validity and ecological relevance of an active in situ bioassay using Gammarus pulex and Limnephilus lunatus. Environ. Toxicol. Chem. 18, 2243-2250.

Shaw, J.L., Manning, J.P., 1996. Evaluating macroinvertebrate population and community level effects in outdoor microcosms: use of in situ bioassays and multivariate analysis. Environ. Toxicol. Chem. 15, 608-617.

Sibley, P.K., Benoit, D.A., Ankley, G.T., 1997. The significance of growth in Chironomus tentans sediment toxicity tests: relationship to reproduction and demographic endpoints. Environ. Toxicol. Chem. $16,336-345$

Sibley, P.K., Benoit, D.A., Balcer, M.D., Phipps, G.L., West, C.W., Hoke, R.A., Ankley, G.T., 1999. In situ bioassay chamber for assessment of sediment toxicity and bioaccumulation using benthic invertebrates. Environ. Toxicol. Chem. 18, 2325-2336.

Soucek, D.J., Cherry, D.S., Currie, R.J., Latimer, H.A., 2000. Laboratory to field validation in an integrative assessment of an acid mine drainage-impacted watershed. Environ. Toxicol. Chem. 19, 1036-1043.

Stuijfzand, S.C., Helms, M., Kraak, H.S., Admiraal, W., 2000. Interacting effects of toxicants and organic matter on the midge Chironomus riparius in polluted river water. Ecotoxicol. Environ. Saf. 46, 351-356.

Taylor, E.J., Maund, S.J., Pascoe, D., 1991. Evaluation of a chronic toxicity test using growth of the insect Chironomus riparius Meigen. In: Jeffrey, D.W., Madden, B. (Eds.), Bioindicators and Environmental Management. Academic Press, London, UK, pp. 343-352.

Tucker, K.A., Burton, G.A., 1999. Assessment of nonpoint-source runoff in a stream using in situ and laboratory approaches. Environ. Toxicol. Chem. 18, 2797-2803.

USEPA, 2000. Methods for Measuring the Toxicity and Bioaccumulation of Sediment-associated Contaminants with Freshwater Invertebrates, second ed. EPA 600/R-99/064.

Watts, M.M., Pascoe, D., 1996. Use of the freshwater macroinvertebrate Chironomus riparius (Diptera: Chironomidae) in the assessment of sediment toxicity. Wat. Sci. Tech. 34, 101-107.

Wentsel, R., McIntosh, A., McCafferty, W.P., 1978. Emergence of the midge Chironomus tentans when exposed to heavy metal contaminated sediment. Hydrobiologia 57 (3), 195-196. 